\title{
AN INVESTIGATION OF THE RELATIONSHIP BETWEEN DISPLACEMENTS OF THE FINGER AND WRIST JOINTS AND THE EXTRINSIC FINGER FLEXOR TENDONS*†
}

\author{
Thomas J. ARMSTRong and Don B. ChafFin \\ Dept. of Industrial Engineering. 2254 Brown Lab. University of Michigan. Ann Arbor. \\ MI 48109, U.S.A.
}

\begin{abstract}
Several investigators have developed biomechanical models of finger flexor tendon displacements during pinching or gripping exertions of hands. Landsmeer has developed the most comprehensive set of models for this purpose. This paper describes experiments in which various sized cadaver hands were used to statistically evaluate the Landsmeer models. In so doing, the effects of hand and wrist anthropometry are included. The results indicate that the tendons displace in relation to joint positions as described by that Landsmeer model in which the tendon is depicted as sliding over the curved articular surface of the proximal bone of the joint. Joint thickness effects were found to modify the parameters in the model as intuitively expected. An empirical prediction model of the anthropometric effects was developed. Further, the tendon displacements for various wrist orientations were expressed empirically for the first time and were shown to be consistent with expected anatomical considerations
\end{abstract}

\section{INTRODUCTION}

Biomechanical models of many aspects of hand function have been developed (Landsmeer, 1960; Dempster, 1961; Smith et al., 1964; Thomas, 1965; Solonen and Hoyer, 1967; Fischer. 1969; Radin, Parker and Paul. 1971; Flatt, 1971; Brand et al., 1975; Chao, Opgrande and Axmear, 1976; Spoor and Landsmeer, 1976). The solutions of these models are dependent on internal dimensions of the hand such as tendon moment arms and tendon displacements. Unfortunately. these dimensions cannot be measured directly in intact hands. Also, though an obviously large variation in hand anthropometry directly affects the parameters in these models, it has not been described rigorously. In addition, the wrist joint generally has been neglected in the existing models. Given these considerations, the objectives of this investigation were defined as follows:

To evaluate biomechanical models of interdigit joint-tendon mechanics that have been proposed by other investigators;

To develop a predictive model of joint and extrinsic finger flexor tendon displacements that can be used for hands of various sizes;

To develop a predictive model of joint and extrinsic finger flexor tendon displacements that can be used for wrists of various sizes.

By achieving these objectives biomechanical models can be used to better investigate desired aspects of hand function and performance for different sized hands.

*27 June 1977: receited for publication 20 September 1977.

† This research was partially supported by an educational gift from AMP. Inc.

\section{Background}

The tendon-joint displacement relationships are determined by the spatial relationships between the tendons and the joints. Three models of tendon-joint displacement have been described by Landsmeer (1960) and will be referred to as Landsmeer's Model I. Model II and Model III.

\section{Model I}

In the first model, the tendon is held securely against the curved articular surface of the proximal bone of the joint (see Fig. 1). During displacement

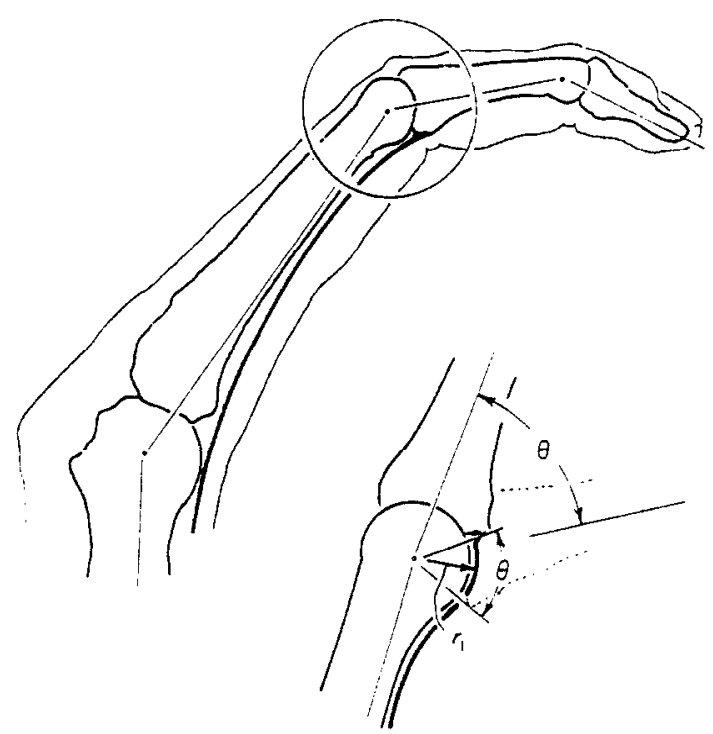

Fig. 1. In Landsmeer's Model I, equation (1), the tendon is held securely against the curved articular surface of the interdigit joints. 


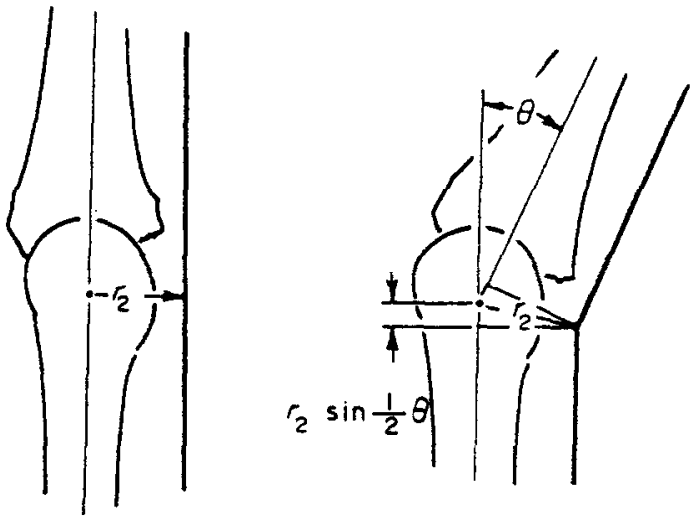

Fig. 2. In Landsmeer's Model II. equation (2). the tendon is securely supported at a point a consiant distance from the joint center.

of the joint. the proximal articular surface can be described as a trochlea. Landsmeer (1960) and Fischer (1969) have shown that the tendon and joint displacement relationship for such a joint is described by the relation:

$$
x=r_{1} \theta .
$$

The tendon moment arm is equal to the perpendicular distance from the joint center to the tendon. In Model I, the center of curvature of the proximal articular surface coincides with the joint center: thus the $r_{1}$ parameter and the tendon moment arm are equivalent (Landsmeer. 1960: Fischer. 1969).

If the tendon is not held securely against the proximal articular surface. it may be displaced away from the joint when the joint is flexed. Landsmeer calls this tendon configuration "bowstringing". This situation is considered in Landsmeer's second and third models.

\section{Model II}

In Landsmeer's second model. the tendon is prevented from bowstringing by a geometric constraint. This configuration is depicted in Fig. 2. In the ideal case, the geometric constraint bisects the included angle of the joint and the tendon resembles two intersecting straight lines. Under these conditions, the tendon and joint displacement are related by the expression:

$$
x=2 r_{2} \sin \left(0_{i} 2\right)
$$

where:

$x=$ the tendon displacement past the joint

$r_{2}=$ the distance from the joint center to the geometric tendon constraint

$0=$ the angle of joint rotation from the neutral position.

For this model the tendon moment arm is the distance from the joint center to the geometric tendon constraint. Thus for Model II the $r_{2}$ parameter and the tendon moment arms are equivalent.

\section{Molel [1]}

It is assumed in Landsmeer's third model that bowstringing exists. This situation is depicted in Fig. 3. Landsmeer (1960) has shown that for a bowstringing tendon. tendon and joint displacement are related as follows:

$$
x=(1)+y(2-() \tan [(1)])
$$

where:

$x=$ the tendon displacement past the joint

$\theta=$ the angle of joint rotation from the neutral position (radians)

$y=$ the distance along the axis of the bone from the point where the tendon begins to curve to the joint center. This distance also equals one half of the tendon arc length when $\theta=0$.

For small angles. $(\theta<40) .(2-0 / \tan (0 / 2))$ is almost linear; for large angles this term introduces some nonlinearity. Based upon this observation. Landsmeer (1960) has stated that Model I might be an acceptable approximation of Model III.
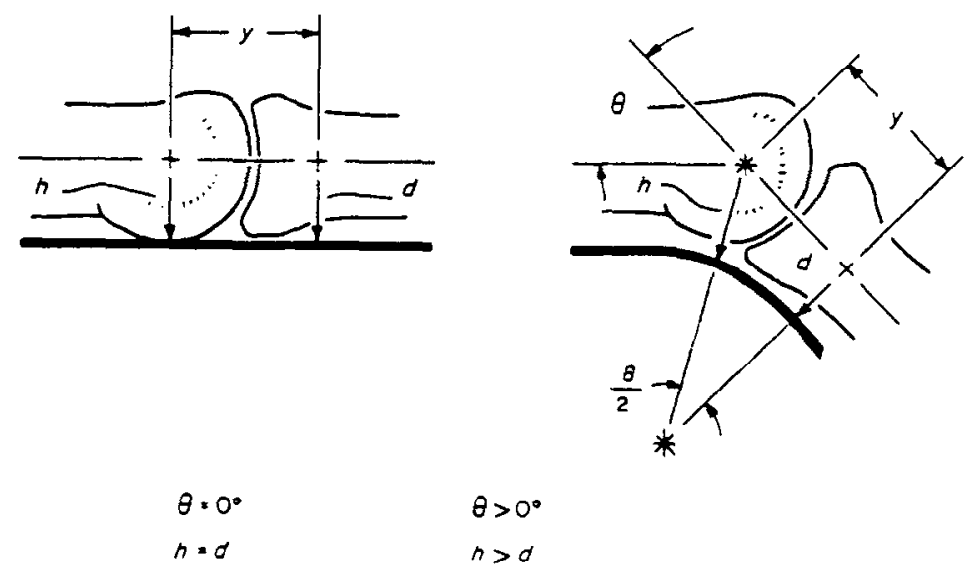

Fig. 3. In Landsmeer's Model III. equation (3). the tendon adjacent to the joint is said to curve or "bowstring." 
The moment arm of a tendon that is permitted to bowstring. $h$. is equal to the distance from the joint center to the tendon along a line between the joint center and the center of the trochlea (see Fig. 3). Landsmeer has shown that the tendon moment arm is calculared as:

$$
h=r\left[\frac{1-\cos (\theta \cdot 2)}{\sin (\theta) 2)}\right]+d .
$$

In all three of Landsmeer's models, the tendon moment arms and tendon displacements are functions of the same parameters.

\section{The wrist model}

When the wrist is flexed, the flexor tendons are supported by the transverse ligaments on the volar side of the carpal tunnel (see Fig. 4a). When the wrist is extended. the flexor tendons are supported by the carpal bones (see Fig. 4b). The tendon-joint displacement relationship is described by equation (1) (Thomas. 1965).

The center of rotation of the wrist joint does not coincide with the origins of the radius of curvature of the structures supporting the extrinsic finger flexor tendons (see Fig. 4). Therefore. the moment arms of the flexor tendons cannot be inferred from observing the tendon joint displacement relationship.

\section{MATERIHLS AND METHODS}

The nature of the study necessitated that the hands be well preserved and that the joints be highly flexible. All of the anatomical material for this investigation was judged to have been healthy in life; hands. wrists, and forearms were severed intact from embalmed cadavers. To determine the effects of hand size. it was necessary that the selected hands include large male and small female hand sizes; joint thickness was used as a measure of hand size. Four suitable embalmed cadaver hands were made available for study by the Department of Anatomy of the University of Michigan. Hands one, two, and four were male and hand three was female. Joint thicknesses of these hands are shown in Table 1; all the corresponding percentile rankings are shown in Table 2. Anthropometric measurements and percentile estimates were based on the methods and data reported by Garrett (1970a and b). Wrist thickness was measured with a sliding caliper at the wrist crease; no estimates were made of the percentile rankings of wrist thickness.

An initial dissection of the forearm was performed to remove tissue not necessary for this study. The flexor digitorum profundus and superficialis tendons were identified, separated from one another, and severed from the muscle approximately two inches proximal to the palmar wrist crease. Except for six inches of the radius, the ulna and the tendons, all tissue proximal to the wrist was removed.

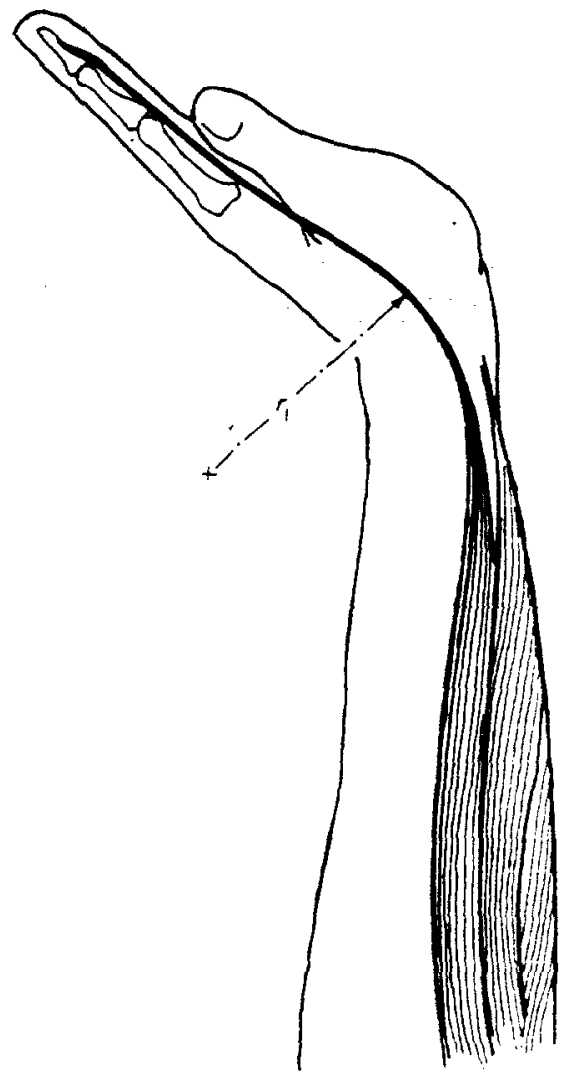

Fig. $4(a)$. In the extended wrist. the extrinsic finger flexor tendons are supported on their dorsal side by the carpal bones.

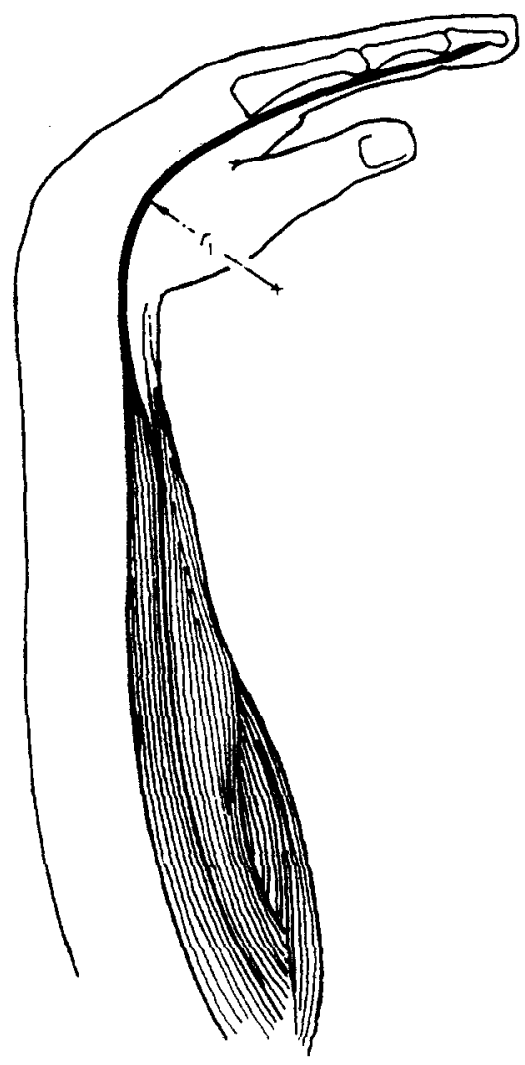

(b). In the flexed wrist, the tendons are supported on their palmar side by the retinacular and volat carpal ligaments. 
Table 1. Interdigital joint thickness of hands used in this investigation expressed in $\mathrm{mm}$

\begin{tabular}{lcrrrr}
\hline & & \multicolumn{5}{c}{ Finger } \\
\multicolumn{1}{c}{ Joint } & Hand & 1 & 2 & 3 & 4 \\
\hline Distul & 1 & 18.5 & 17.0 & 15.0 & 14.5 \\
Interphalangeal & $?$ & 16.5 & 17.0 & 16.0 & 14.5 \\
& 3 & 9.0 & 10.0 & 9.5 & 8.5 \\
& 4 & 14.5 & 17.0 & 15.0 & 12.5 \\
Proximal & 1 & 21.5 & 22.0 & 19.5 & 19.0 \\
Interphilangeal & 2 & 21.5 & 22.0 & 21.5 & 17.0 \\
& 3 & 11.5 & 12.5 & 12.0 & 10.5 \\
& 4 & 17.0 & 19.5 & 17.5 & 14.5 \\
Metacurpal & 1 & 26.5 & 27.0 & 23.5 & 20.5 \\
Philiangeal & 2 & 25.0 & 26.5 & 22.5 & 21.0 \\
& 3 & 18.0 & 19.0 & 18.0 & 15.5 \\
& 4 & 25.5 & 26.0 & 24.0 & 20.0 \\
Wrist & 1 & 45.5 & & & \\
& 2 & 35.5 & & & \\
& 3 & 30.0 & & & \\
\hline
\end{tabular}

The hands were secured to a stiff steel frame as shown in Fig. 5. The tendons were attached one at a time during the experiment to a displacement micrometer via a clamp. The micrometer was equipped with a strain ring that indicated the tension on the tendon. A constant 19.6 $\mathrm{N}$ tension was maintamed on the tendon for all measurements. Using the value of Young's Modulus reported by Harris 119661. $7.6 \times 10^{8} \mathrm{P}$. the strain for a tendon with a crosssectional area of $2.5 \mathrm{~mm}^{2}$ for a $2 \mathrm{~N}$ load is less than $I^{\prime \prime}$, "

All tendon and joint displacements were measured from a neutral position: i.e. that position where the long axes of the articulating bones are essentially parallel. The tendons were displaced in $2.5 \mathrm{~mm}$ increments from this neutral position while a $2 \mathrm{~N}$ load was maintained: joint positions were recorded photographically. Successive tendon and joint positions were recorded throughout the range of motion: this procedure was followed twice for each joint. Between 8 and 15 data points were recorded for each joint. Visually detected radial and ulnar deviations were corrected as was necessary so that all tendon and joint displacements were for flexion and extension in one plane.

Table 2. Percentile ratings of the distal and proximal interphalangeal joints for the hands used in this study /Garrett 1970a.b)

\begin{tabular}{lccccc}
\hline \multicolumn{1}{c}{ Joint } & Subject & \multicolumn{4}{c}{ Finger } \\
No. & 1 & 2 & 3 & 4 \\
\hline Distal & 1 & 99 & 80 & 50 & 75 \\
Interphalangeal & 2 & 80 & 80 & 80 & 75 \\
& 3 & $<1$ & $<1$ & $<1$ & $<1$ \\
& 4 & 25 & 80 & 50 & 16 \\
Proximal & 1 & 94 & 89 & 67 & 91 \\
Interphalangeal & 2 & 94 & 89 & 97 & 31 \\
& 3 & $<1$ & $<1$ & $<1$ & $<1$ \\
& 4 & 3 & 36 & 12 & 3 \\
\hline
\end{tabular}

For measurement of wrist joint displacements. the four fingers were splinted together so that the hand moved as a single unit. The tendon joint displacement relationship was obsersed for each of the profundus and superficialis tendons.

Before data on the metacarpal-phalangeal joint were taken. the palm of the hand was dissected and the profundus tendons were separated inside the carpal tunnel. After all of the measurements were taken for a metacarpal-phalangeal joint. the corresponding finger was removed to allow an unobstructed view of the adjacent finger. This procedure was repeated until all of the four fingers were removed.

The separate fingers then were secured individually to the apparatus to measure tendon and joint displacements at the proximal and distal interphalangeal joints. To prevent its displacement while the proximal interphalangeal joint was studied. the distal interphalangeal joint was made rigid with a splint.

\section{RESLLTS}

Tendon-joint displacement data were obtained from four hands for a total of sixteen different distal interphalangeal. proximal interphalangeal and metacarpal joints and a total of four wrist joints. By obsersing elght to fifteen tendon-joint positions for each tendon at each joint. a total of 976 observations was obtuined for the finger joints and a total of 737 observations was obtained for the wrist joints. Those data for which it appeared that the joints might have been displaced beyond the normal range of motion were deleted.

Landsmeer's Model I was selected as the best model on the basis of hou well each model fit the observed tendon-joint displacement and on the soundness of the underlying assumptions of each model. To determine which model fit best. equations $\{2-4\}$ were fitted by the method of least squared errors to the tendonjoint displacement data for each joint of each finger of each subject as shown in Fig. 6. The $r_{1}$ parameter of equation (1). the $r_{2}$ parameter of equation (2). and the $d$ and $y$ parameters of equation (4) were estimated in a total of thirty-two cases for the flexor digitorum superficialis. Based on an $F$ test of the regression and residual variances. all of the relationships were determined to be significant at $x \leq 0.0001$. Landsmeer s first (wo models. equations (1) and (2), contain only one parameter: therefore. the significance of the relationship also applies to the $r_{1}$ and $r_{2}$ parameter estimates. The boustring model. equation (3), is a two parameter model: therefore? statistics were calculated for each estimate of the $d$ and $y$ parameters. In all cases the $d$ parameter was significant at $x \leq 0.05$, i.e. $d>0$.

The y parameter was significantly greater than zero. at $x \leq 0.05$. in only seventeen of the forty-eight cases for the flexor digitorum profundus and was significantly greater than zero in only seven of the thirtytwo cases for the flexor digitorum superficialis. 


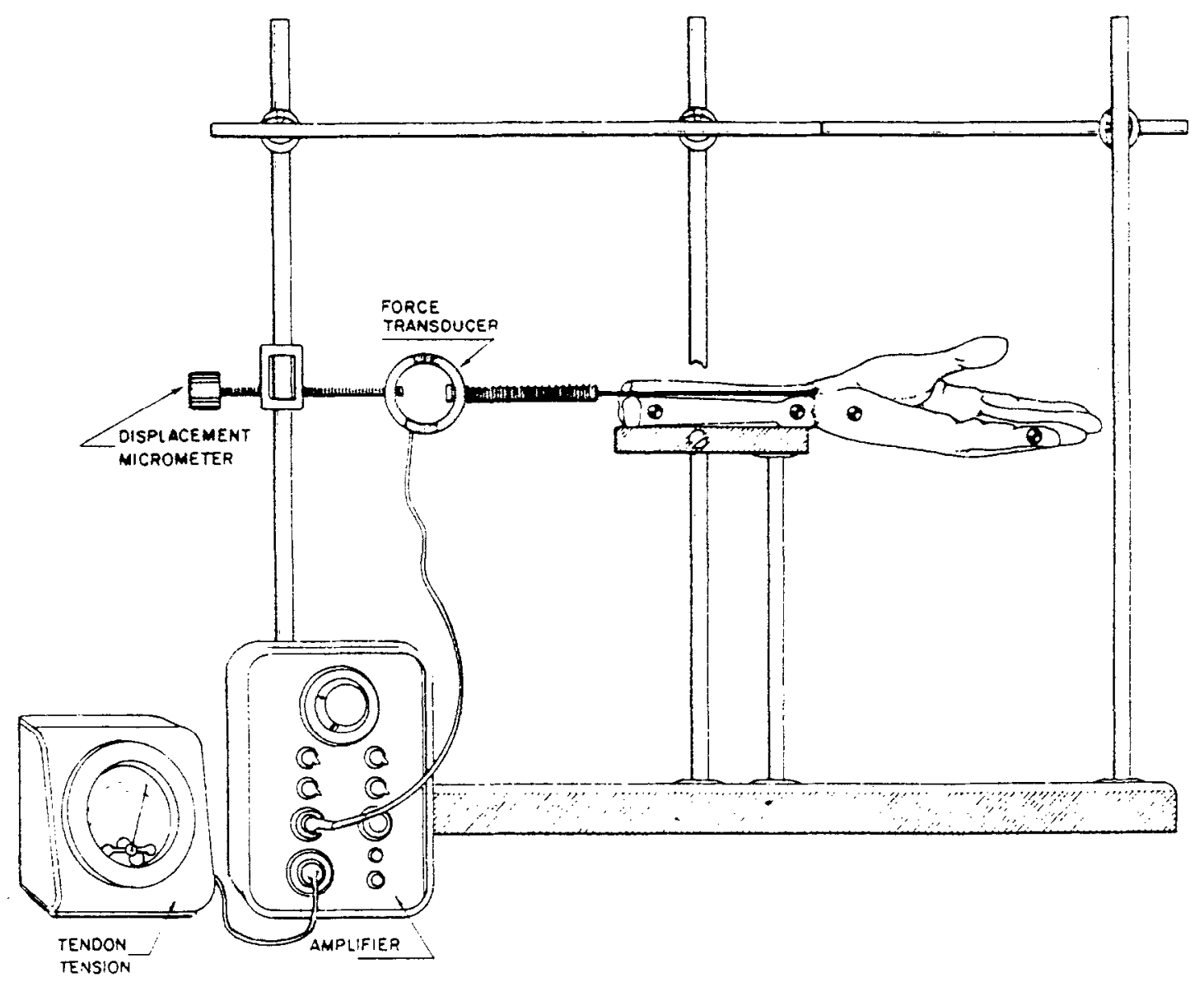

Fig. : Tendon and joint displacements were measured on an apparatus as shown. A force transducer was located between the tendon and the displacement micrometer so that the tendon load could be kept constant during all measurements.

Cases for which $y$ is less than zero are at variance with the tendon-joint geometry postulated for :Model III: cases for which $y$ is equal to zero are equivalent to Model 1. Based upon the large number of cases for which the parameter estimates of $y$ are less than or equal to zero. Landsmeer's bowstring model was rejected as a usable model of the tendon-joint displacement relationship for intact joints.

To test the relative goodness of fit of Models 1 and II to the data. the residual errors of the models were compared for each joint of each finger of each hand. Model I accounted for a greater portion of the observed tendon displacement variance more frequently than did Model II in twenty-seven of fortyeight cases for the profundus tendon and in twenty of thirty-two cases for the superficialis tendon. However. none of these differences was statistically significant at $x \leq 0.05$.

Finally. Model I appeared to be based on the most creditable assumption-that the tendon is displaced over a trochlea such as the curved surface of the proximal bone of the joint or the bulging palmar plate isee Fig. 11. It is likely that the tendon is held against the trochlear structure by compression of pal- mar tissuz and external load forces adjacent to the joints. Longitudinal cross-sectional photographs of the interdigit joints showed that the tendons closely follow the phalanges and did not show tendon bowstringing (Landsmeer. 1976 ).

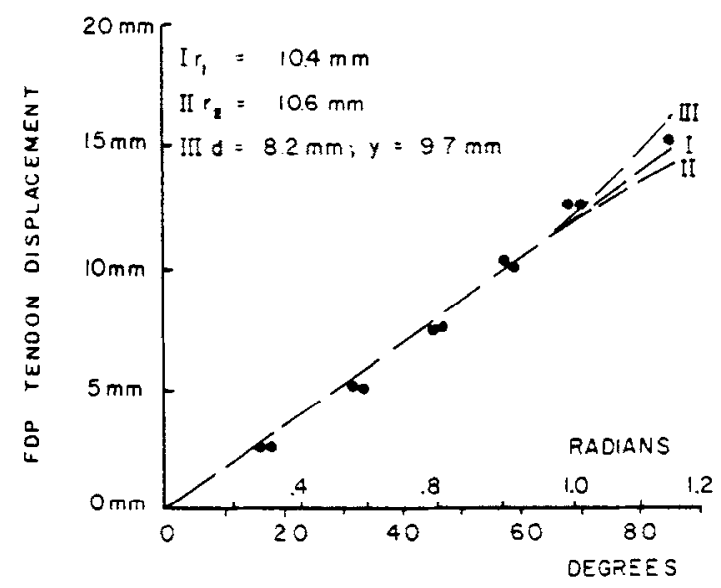

Fig. 6. The parameters in Landsmeer's squared error for each Model I. II. and III were estimated by the method of least combination of subject. finger. joint. and tendon. 
Model II is based on the assumption that the tendon forms intersecting straight lines and that the intersection bisects the included joint angle (see Fig. 2). This assumption has not been validated; the human body seldom tends to be straight or symmetrical.

The second objective of this investigation was to develop a predictive model of the tendon-joint displacement relationship for various sized finger joints. The pooled tendon-joint displacement data for all subjects are shown for each tendon-joint combination in Fig. 7. The following empirical model was fitted to the tendon-joint displacement data by the method of least squared errors:

Tendon displacement $(\mathrm{mm})=0.1034 X_{5}$

$$
\begin{array}{r}
+0.004211 X_{4} X_{3}-0.0162 X_{3} X_{3}-0.03043 X_{1} X_{3} \\
-0.06818 X_{2} X_{3}+0.03679 X_{1} X_{3} X_{3},
\end{array}
$$

where:

$X_{1}=1$ for the proximal interphalangeal joint: 0 for other joints

$X_{2}=1$ for the distal interphalangeal joint; 0 for other joints

$X_{3}=1$ for the profundus tendon; 0 for the superficialis

$X_{4}=$ joint thickness $(\mathrm{mm})$

$X_{3}=$ joint angle (degrees from straight finger).

The fitted model accounted for $96^{\circ} \%$ of the observed variance of the tendon displacement and was significant at $\alpha \leq 0.01$; the standard error of regression was $0.86 \mathrm{~mm}$. Equation (5) can be reduced to the same form as equation (1) by inserting the appropriate values of $X_{1}, X_{2}, X_{3}$ and $X_{4}$ and adjusting the resulting coefficient for radians instead of degrees.

Equation (5) was tested to determine if it accounted for all differences among the tendon-joint displacement relationships of different hands and fingers. The only significant hand or finger difference, at $\alpha \leq 0.05$, was found between tendon displacements for hands two and one; however, the difference between these two hands was found to be small-less than $0.8 \mathrm{~mm}$ of the tendon displacement per $100^{\circ}$ of joint rotation. Consideration of this difference between hands reduced the unexplained variance of equation (5) by only $6.9 \%$ and reduced the standard error of the regression from 0.92 to $0.88 \mathrm{~mm}$. The unexplained variance among hands can be neglected with only a small effect on the precision of finger model predictions.

A second and more mechanistic approach for predicting tendon displacements is first to estimate $r_{1}$ and then to calculate tendon displacement using equation (1). The estimates of $r_{1}$ for each tendon-joint combination are shown plotted against joint thickness in Fig. 8. The following model was fitted to these data:

$$
\begin{aligned}
r_{1}(\mathrm{~mm})= & 6.19-1.66 X_{1}-4.03 X_{2} \\
& -0.77 X_{3}+1.76 X_{1} X_{3}+0.225 X_{4} .
\end{aligned}
$$

where $X_{1}, X_{2}, X_{3}$, and $X_{4}$ have the same meaning as for equation (5).

Equation (6) accounted for $92^{\circ}$ of the observed variance of $r_{1}$ and was significant at $x \leq 0.01$ : the standard error of the regression was $0.75 \mathrm{~mm}$.

The third objective of this investigation was to develop a predictive model of extrinsic finger flexor tendon-wrist joint displacement for wrists of different sizes. The pooled tendon-joint displacement data for all subjects are shown for both tendons in Fig. 9. The following model was fitted by the method of least squared errors to these data:

Tendon displacement $(\mathrm{mm})=0.0263 W_{1}+$

$$
\begin{aligned}
0.0050 W_{1} W_{2} & +0.106 W_{1} W_{3} \\
& -0.000960 W_{1} W_{2} W_{1} .
\end{aligned}
$$

where:

$W_{1}=$ joint angle in degrees from neutral wrist position $\left(W_{1}>0\right.$ wrist flexion; $W_{1}<0$ wrist extension)

$W_{2}=$ joint thickness $(\mathrm{mm})$

$W_{3}=0$-extension; 1 -flexion

$W_{4}=0$-superficialis; 1 -profundus.

The fitted model accounted for $97 \%$ of the tendon displacement variance and was significant at $z \leq 0.01$; the standard error of the regression was $1.52 \mathrm{~mm}$. Equation (7) reduces to the same form as equation (1) when the appropriate values are inserted for $W_{2}, W_{3}, W_{4}$ and the resulting coefficient is adjusted for radians.

Equation (7) was tested to determine if it accounted for all differences among tendon-joint displacement relationships of different hands and fingers. Displacement differences among all fingers and between hands one and two were found to be significant at $x \leq 0.05$. Differences among the fingers were less than $2.8 \mathrm{~mm}$ per $100^{\circ}$ of wrist movement; inclusion of this difference among fingers reduced the unexplained variance by only $6 \%$. The standard error of regression of the wrist model was reduced to $1.48 \mathrm{~mm}$.

The displacement difference between hand one and two, the thickest and the thinnest male hands, was $5.8 \mathrm{~mm}$ per $100^{\circ}$ of wrist movement. Consideration of this difference between hands reduced the explained variance associated with equation (7) by $21 \%$ : the associated standard error of the regression was reduced to $1.35 \mathrm{~mm}$. Differences between hands and fingers can be neglected with only a small sacrifice in the precision of equation (7).

\section{DISCUSSION}

The major findings of this investigation are that Landsmeer's Model I is the best description of interdigit tendon-joint mechanics and that the $r_{1}$ parameter in this model is related to hand size. Although embalmed hands were used in the investigation, no reasons are seen that the findings should not be used 

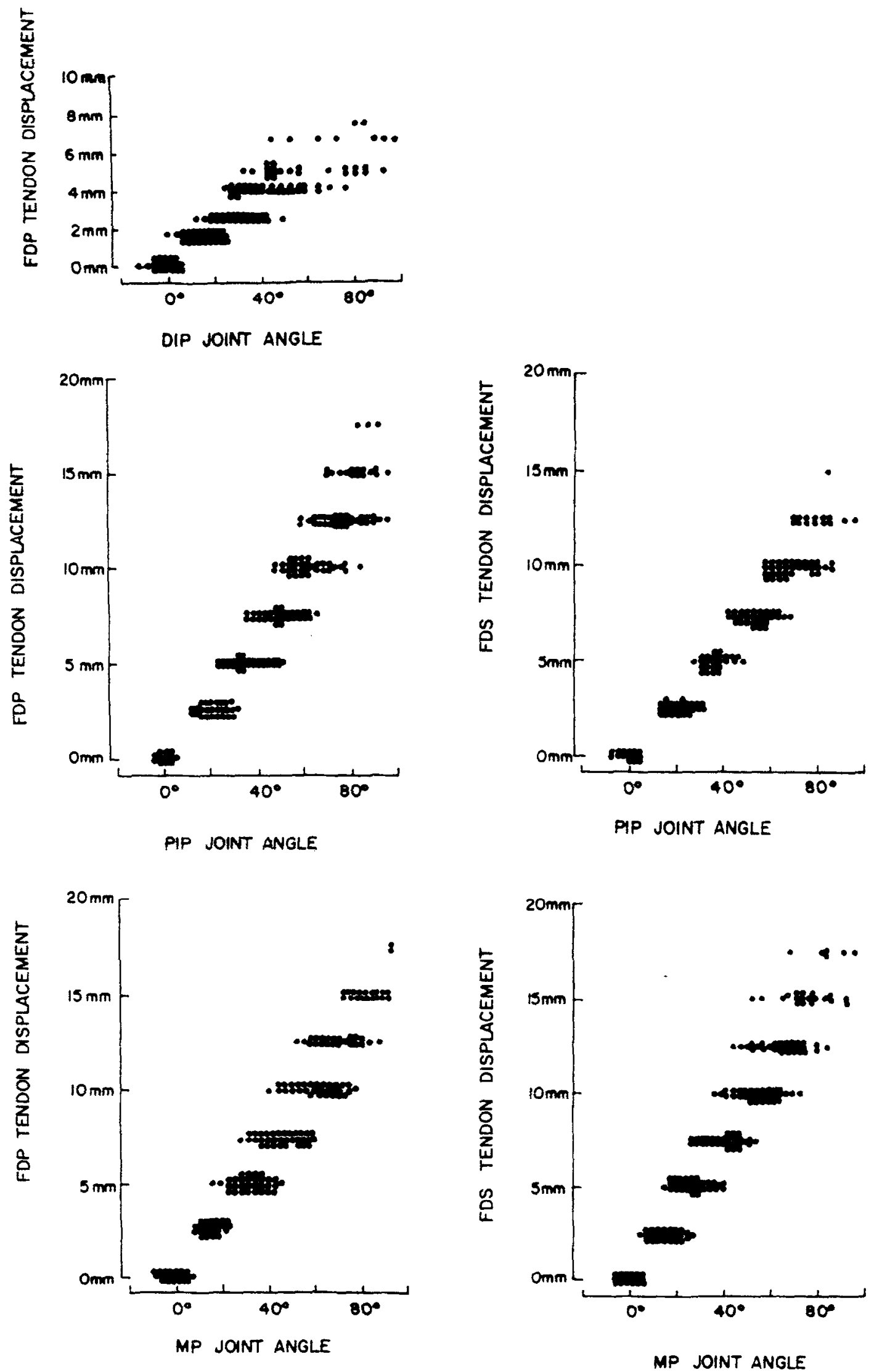

Fig. 7. The tendon-joint displacement data for the profundus and superficialis tendons at metacarpalphalangeal (MP). the proximal interphalangeal (PIP), and the distal interphalangeal joints (DIP). pooled for all four hands. are shown. 


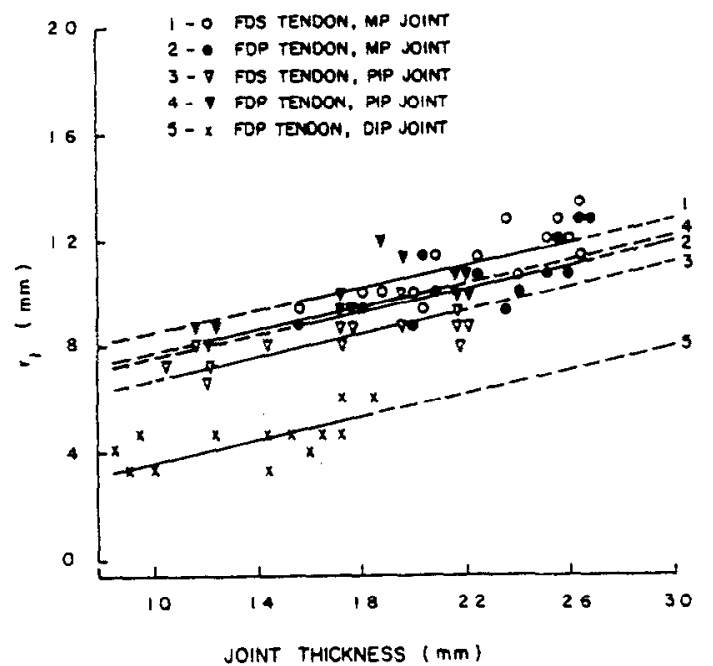

Fig. 8. The trochlear radii vs joint thickness and filted regression lines [equation (7)] for the flexor digitorum profundus and superficialis at the distal and proximal interphalangeal and metacarpal-phalangeal joints are shown above. The solid lines denote the range of the observed joint thick ness.

to describe the behavior of living hands. The findings are based on the mechanical behavior of the relatively inert tissues of the hand-bone, tendons, and ligaments-none of which has been shown to undergo any important mechanical changes with embalming.

Based on the sizes of male and female hands reported by Garrett $(1970 \mathrm{a}, \mathrm{b})$ the predicted tendon displacements do not vary as much among different hands, fingers and tendons as among different joints. The predicted flexor digitorum profundus and superficialis tendon displacements [equation $(15)$ ] past the distal and proximal interphalangeal and the metacarpalphalangeal joints for the long finger of a 5 percentile female and a 95 percentile male (Garrett, 1970a, b) are listed in Table 3. These predictions encompass $95^{\circ} \%$ of the combined male and female populations. Profundus and superficialis tendon displacements for the small female and the large male hands differ by as much as $4.8 \mathrm{~mm} / 100^{\circ}$ of metacarpal-phalangeal joint rotation and by as little as $2.7 \mathrm{~mm} / 100^{\prime}$ of distal interphalangeal joint rotation: in all cases the tendon displacements are greater for the large hands than for the small hands. For the large male and small female hands respectively, profundus tendon displacements are 14.4 and $12.3 \mathrm{~mm} 100$ of joint rotation greater at the metacarpal-phalangeal joint than at the distal interphalangeal joint : displacements are 5.1 and $3.5 \mathrm{~mm} / 100$ of the joint rotation greater at the metacarpal-phalangeal joint than at the proximal interphalangeal joint. Similarly. superficialis tendon displacements are 8.7 and $7.1 \mathrm{~mm} / 100$ of joint rotation greater at the metacarpal-phalangeal joint than at the proximal interphalangeal joint.

For both the 5 percentile female and the 95 percentile male sizes. predicted tendon displacements at the proximal interphalangeal joint are $2 \mathrm{~mm} / 100^{\circ}$ greater for the flexor digitorum profundus than for the superficialis. At the metacarpal-phalangeal joint. the tendon displacement is $1.6 \mathrm{~mm}: 100^{\circ}$ greater for the superficialis than for the profundus. Thus. tendon displacements vary less among different tendons than among different joints or different hands.

The predictions shown in Table 3 also are significant in that they are consistent with the anatomical arrangement of the tendons and joint centers. The $r_{1}$ parameters of equation (1). predicted with equation (6) for the long finger of a 5 percentile female and a 95 percentile male, are shown in Table 3. At the proximal interphalangeal joint of the 5 percentile female and the 95 percentile male. the predicted $r_{1}$ values of the profundus tendons are $1.0 \mathrm{~mm}$ greater than those of the superficialis. At the metacarpal-phalangeal joint of the 5 percentile female and 95 percentile male, the predicted values of $r_{1}$ for the profundus tendon are $0.8 \mathrm{~mm}$ less than those for the superficialis. At the proximal interphalangeal joint. the superficialis tendon is closer to the joint center than is the profundus; at the metacarpal-phalangeal joint, the profundus tendon is closer to the joint center than is the superficialis. In the course of this study, the profundus and superficialis tendons were observed to be approx. $1 \mathrm{~mm}$ thick. This arrangement of the tendons accounts for the differences in the rates of tendon displacements and the differences in the predicted $r_{1}$ values between the metacarpal-phalangeal and the proximal interphalangeal joints [equation (5)].

The predicted flexor digitorum profundus and

Table 3. Joint thicknesses (Garrett. 1970a. 1970b). predicted tendon displacement per 100 of joint rotation [equation (5)]. and predicted values of $r$ [equation (6)]. for a 5 percentile female and a 95 percentile male

\begin{tabular}{|c|c|c|c|c|c|c|}
\hline Joint & Population & $\begin{array}{c}\text { Joint } \\
\text { thickness }\end{array}$ & $\begin{array}{l}\text { Profur } \\
\text { Tendon } \\
\text { displacement }\end{array}$ & $r_{1}$ & $\begin{array}{l}\text { Superfi } \\
\text { Tendon } \\
\text { displacement }\end{array}$ & $r_{1}$ \\
\hline $\begin{array}{l}\text { Distal } \\
\text { Interphalangeal }\end{array}$ & $\begin{array}{c}5^{\circ} \text {. Female } \\
95^{\circ} \text {. Male }\end{array}$ & $\begin{array}{l}11.6 \mathrm{~mm} \\
18.1 \mathrm{~mm}\end{array}$ & $\begin{array}{l}6.8 \mathrm{~mm} / 100 \\
9.5 \mathrm{~mm} / 100\end{array}$ & $\begin{array}{l}4.0 \mathrm{~mm} \\
5.5 \mathrm{~mm}\end{array}$ & - & - \\
\hline $\begin{array}{l}\text { Proximal } \\
\text { Interphalangeal }\end{array}$ & $\begin{array}{l}5^{\circ}{ }_{\circ} \text { Female } \\
95^{\circ}, \text { Male }\end{array}$ & $\begin{array}{l}14.9 \mathrm{~mm} \\
22.5 \mathrm{~mm}\end{array}$ & $\begin{array}{l}15.6 \mathrm{~mm} / 100^{\circ} \\
18.8 \mathrm{~mm} / 100^{\circ}\end{array}$ & $\begin{array}{r}8.9 \mathrm{~mm} \\
10.6 \mathrm{~mm}\end{array}$ & $\begin{array}{l}13.6 \mathrm{~mm} / 100 \\
16.8 \mathrm{~mm} / 100\end{array}$ & $\begin{array}{l}7.9 \mathrm{~mm} \\
9.6 \mathrm{~mm}\end{array}$ \\
\hline $\begin{array}{l}\text { Metacarpal- } \\
\text { phalangeal }\end{array}$ & $\begin{array}{l}5_{\circ}^{\circ} \text { Female } \\
95^{\circ} \text { Male }\end{array}$ & $\begin{array}{l}24.6 \mathrm{~mm} \\
36.1 \mathrm{~mm}\end{array}$ & $\begin{array}{l}19.1 \mathrm{~mm} / 100 \\
23.9 \mathrm{~mm} / 100\end{array}$ & $\begin{array}{l}11.0 \mathrm{~mm} \\
13.5 \mathrm{~mm}\end{array}$ & $\begin{array}{l}20.7 \mathrm{~mm} / 100 \\
25.5 \mathrm{~mm} / 100\end{array}$ & $\begin{array}{l}11.7 \mathrm{~mm} \\
14.3 \mathrm{~mm}\end{array}$ \\
\hline
\end{tabular}



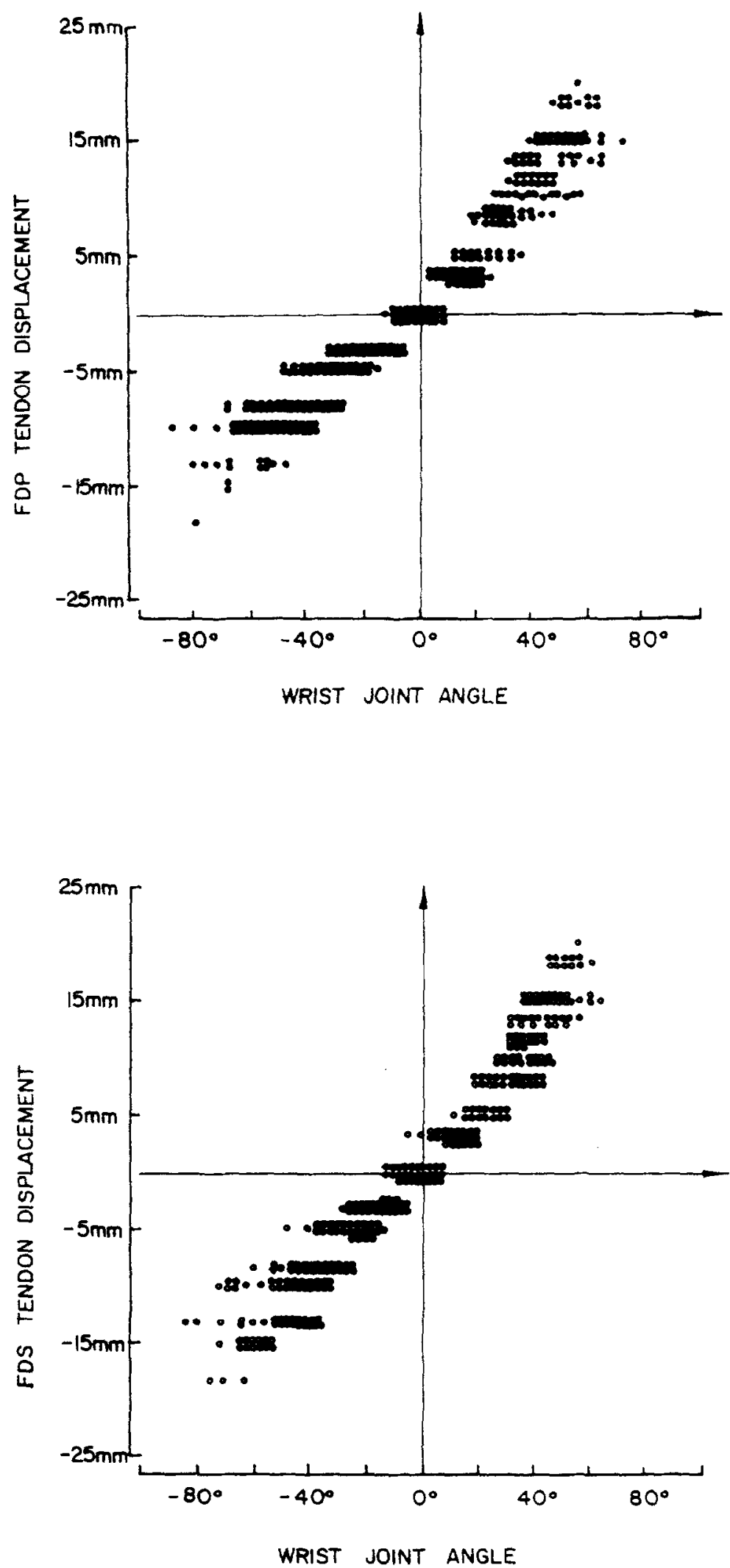

Fig. 9. The tendon-joint displacement data for the profundus and superficialis tendons at the wrist joint, pooled for all four hands. are shown. 
Fig. 4. Estimated wrist joint thicknesses (Garrett. 1970) and predicted tendon displacements per $100^{\circ}$ of joint rotation (equation (7))

\begin{tabular}{lccccc}
\hline & & \multicolumn{2}{c}{ Tendon displacement $\left(\mathrm{mm} ; 100^{\circ}\right)$} \\
$\begin{array}{c}\text { Estimated } \\
\text { wrist thickness } \\
(\mathrm{mm})\end{array}$ & Profundus & \multicolumn{2}{c}{ Superficialis } \\
Population & 31.8 & -15.6 & 26.2 & -18.7 & 29.3 \\
\hline $5 \%$ Female & 44.8 & -21.0 & 31.6 & -25.2 & 35.8 \\
\hline $95 \%$ Male & & Extension & Flexion & Extension & Flexion \\
\hline
\end{tabular}

superficialis tendon displacements past the wrist joints for an estimated* 5 percentile female and 95 percentile male wrist thickness are listed in Table 3 [equation (7)]. This table shows that the tendon displacement caused by wrist flexion or extension is greater for the flexor digitorum superficialis tendon than for the profundus. When the wrist is extended, the superficialis tendon displacement is $4.2 \mathrm{~mm} / 100^{\circ}$ for the large male hand and $3.1 \mathrm{~mm} / 100^{\circ}$ for the small female hand greater than the corresponding profundus tendon displacement. From equation (2) it can be shown that the radius of tendon curvature in the wrist is greater for the superficialis than for the profundus. This result is consistent with the spatial arrangement of the tendons in the wrist; when the wrist is extended, the superficialis tendons are supported by the profundus tendons, which in turn are supported by the carpal bones. Thus, the radius of tendon curvature would be greater for the superficialis tendons than for the profundus tendons. Also by this argument it would be expected that for a given angle of wrist flexion, the profundus displacement would be greater than the superficialis displacement; however, the predicted superficialis tendon displacement is greater than the profundus displacement (Table 4). When the wrist is flexed, the superficialis tendon displacement is $4.2 \mathrm{~mm} / 100^{\circ}$ for the large hand and $3.1 \mathrm{~mm} / 100^{\circ}$ for the small hand greater than the profundus displacement. This small inconsistency probably is due to differences in the paths of the tendons in or near the carpal tunnel. Future research is required to determine the geometric relationship between the extrinsic finger flexor tendons and the anatomical structures of the wrist.

It is hoped that these findings will be useful in applying existing biomechanical models. As noted in the background section of this paper, the tendon moment arm is the $r_{\mathrm{l}}$ parameter in Landsmeer's first model; therefore these results could be used in analyses of hand-tendon forces as well as of tendon dis-

* Wrist thicknesses were estimated from standard wrist circumference and wrist bread th data (Garrett, 1970), using the formula for the circumference of an ellipse:

$$
c=\pi \sqrt{\left(a^{2}+b^{2}\right) / 2} \text {. }
$$

placements. Future research might also include validation of these findings via radiograph of joints.

\section{REFERENCES}

Brand, P. W. Cranor, K. C. and Ellis. J. C. (1975) Tendon and pulleys at the metacarpal joint of a finger. $J$. Bone Jnt. Surg. 57-A, 779-784.

Chao, E. Y., Opgrande. J. D. and Axmear, F. E. (1976) Three-dimensional force analysis of finger joints in selected isometric hand functions. J. Biomechanics 9 , 387-396.

Dempster, W. T. (1961) Free body diagrams as an approach to the mechanics of human posture and motion. In Biomechanical Studies of the Musculo-skeletal Sistem (edited by Evans et al.). Charles C. Thomas, Springfield. IL.

Fischer, G. W. (1969) A Treatise of the Topographical Anatomy of the Long Finger and a Biomechanical Investigation of Its Interjoint Motement. Doctoral dissertation in Engineering Mechanics, the University of Iowa, IA.

Flatt, A. E. (1971) The pathomechanics of ulnar drift. Social Rehabilitation Services, Final Report, Grant No. RD 2226M.

Garrett. J. W. (1970a) Anthropometry of the Hands of Male Air Force Flight Personnel. Aerospace Medical Research Laboratory, Aerospace Medical Division. Air Force Sys. tems Command, Wright-Patterson Air Force Base, OH

Garrett, J. W. (1970b) Anthropometry of the Air Force Female Hand. Aerospace Medical Research Laboratory, Aerospace Medical Division, Air Force Systems Command, Wright-Patterson Air Force Base, OH.

Harris, E. H., Walker, L. B. and Bass, B. R. (1966) Stressstrain studies in cadaveric human tendon and an anomaly in the Young's modulus thereof. Med. Biol. Engng 4, 253-259.

Landsmeer, J. M. F. (1960-62) Studies in the anatomy of articulation. Acta Morph. Neerlands scand. 3-4,

Landsmeer, J. M. F. (1976) Atlas of Anatomy of the Hand. Churchill Livingston, p. 246.

Radin, E. L., Parker, H. G. and Paul, I. L. (1971) Pattern of degenerative arthritis, preferential involvement of distal finger-joint. Lancet 1, 377.

Smith. E. M., Juvinall. R. C.. Bender. L. F, and Pearson. J. R. (1964) Role of the finger flexors in rheumatoid deformities of the metacarpal-phalangeal joints. Arth. Rheum. 7, 467-480.

Solonen. K. A. and Hoyer. P. (1967) Positioning of the pulley mechanism when reconstructing deep flexor tendons of the fingers. Acta orthop. scand. 38, 321-328.

Spoor, C. W. and Landsmeer. J. M. F. (1976) Analysis of the zigzag movement of the human finger under the influence of the extensor digitorum and the deep flexor tendon. J. Biomechanics 9, 561-566.

Thomas. D. H. (1965) The Phisical Properties of the Human Finger, PhD thesis, Case Institute of Technology. University Microfilms. Ann Arbor, MI 